Doi: $10.15863 /$ TAS

\section{International Scientific Journal}

\section{Theoretical \& Applied Science}

p-ISSN: 2308-4944 (print) e-ISSN: 2409-0085 (online)

Year: $2014 \quad$ Issue: 12 Volume: 20

Published: $30.12 .2014 \quad$ http://www.T-Science.org
Georgy Vasil'evich Tokmazov

Associate professor,

Candidate of Pedagogical Science,

Professor Department of Mathematics, State Maritime University Admiral Ushakov, Russia

tokmazov@mail.ru

SECTION 21. Pedagogy. Psychology. Innovation in Education.

\title{
MATHEMATICAL MODELING OF RESEARCH SKILLS METHODS OF PETRI NETS
}

Abstract: The mathematical modeling research skills in educational activity methods of Petri nets based on generalized integral-cognitive actions taking into account the multi-dimensional didactic and mathematical processes and activities with respect to substantive components of cognitive processes.

Key words: mathematical modeling, Petri nets, research skills, objectivity, activity, generally, teaching and research competence.

Language: Russian

Citation: Tokmazov GV (2014) MATHEMATICAL MODELING OF RESEARCH SKILLS METHODS OF PETRI NETS. ISJ Theoretical \& Applied Science 12 (20): 42-46. http://dx.doi.org/10.15863/TAS.2014.12.20.10

\section{УДК 372.851}

\section{МАТЕМАТИЧЕСКОЕ МОДЕЛИРОВАНИЕ ИССЛЕДОВАТЕЛЬСКИХ УМЕНИЙ МЕТОДАМИ СЕТЕЙ ПЕТРИ}

Аннотация: Предложено математическое моделирование исследовательских умений в учебной деятельности методами сетей Петри на основе обобщеённо-иелостных познавательных действий с учётом многомерных дидактико-математических процессов относительно предметных и деятельных компонентов познавательных прочессов.

Ключевые слова: математическое моделирование, сети Петри, исследовательские умения, предметность, деятельность, обобщённость, учебно-исследовательские компетенции.

\begin{abstract}
Дальнейшее математического развитие процесса обобщённых деятельностных основ, целостного представления структур и дидактики формирования знания исследовательских умений ориентируется на применение математических форм сетей Петри. Сети Петри отражают математическое и графическое моделирование различных познавательных процессов обобщённой исследовательской деятельности. Данные программные средства являются дидактическим информационным методом преобразования учебной информации относительно подготовки образовательных элементов: стохастических управляемых систем обработки познавательной исследовательской
\end{abstract}

информации. Сети Петри отражают графическое представление обобщённой исследовательской деятельности относительно всеобщего процесса развития в условиях подготовки универсального учебного субъекта. Данные сети позволяют создавать интегральные ядра графологических элементов, позволяющих моделировать интериоризационные и экстериоризационные циклы образовательной деятельности на рефлексивной основе $[1 ; 2]$.

Математическое моделирование исследовательских умений методами сетей Петри позволяет: составлять уравнения состояния исследовательской деятельности относительно обобщённого представления всего процесса познания; формировать алгебраические уравнения развития 
исследовательских действий и самого математического предмета исследования; устанавливать динамику адаптивного прогнозирования аналитической деятельности. Сети Петри управляют процессами: организации коллективных форм исследовательских познавательных умений; проектирования методологических моделей дидактико-математического моделирования нормативной творческо-исследовательской деятельности; составлять систему инвариантных форм учебно-исследовательских навыков; создавать схемы учебно-производственных исследовательских состояний. Сети Петри позволяют организовать макро- и микроисследовательский процесс дидактикоматематического моделирования. Для построения системы математического моделирования применяются общелогические методы проектирования относительно психолого-дидактической теории деятельности, целостного процесса познания и технологии формирования интеллекта [ 3,4$]$.

Построенная дидактико-математическая система исследовательских процессов моделируется сетью Петри методом общей схемы исследовательской деятельности в данных условиях с учётом субъектных отношений процесса производства исследовательского умения. При этом анализируются внешние параметры исследовательского умения, его структура относительно базисных исследовательских компонентов и познавательных отношений при любом уровне учебной деятельности. Возникающие локальные дидактические риски указывают на нарушение общей схемы построения исследовательского процесса. Это приводит к полному обобщённому контролю процесса проектирования исследовательского умения графо-аналитическими моделями сетей Петри. Исследовательский процесс, в данном случае, отражает общую схему структурного процесса построения исследовательских ячеек Петри относительно всего исследовательского пространства. Данный этап позволяет организовать многофазное модифицированное проектирование исследовательского умения относительно целостного представления субъектного развития образа Мира. Создание абсолютного исследовательского смыслообразования формирует общую логику целостного исследовательского процесса, материальным носителем которого и являются сети Петри [ $5 ; 6]$.

Циклический характер математического проектирования исследовательских умений сетью Петри позволяет выделить три основных фазы, которые отражают общую структуру микроанализа исследовательского действия: ориентировочную, исполнительную и контрольную компоненты. Выделенные составные элементы процесса исследования имеют собственную структуру относительно всего процесса производства знания в заданных параметрах. Существенное значение имеет ориентировочный образ исследовательского умения, который формируется до насыщения целостностью на каждом этапе формирования познавательного образа и смыла его существования и развития. Исполнительная часть исследовательского образа отражает динамику состояния сети Петри в данный момент исследовательского исполнения. Контрольная часть исследовательского состояния определяет динамику соответствия результативного и проективного образов исследовательского умения, что позволяет сетями Петри проводить мгновенную коррекцию процесса познания. Это определяет условия целостного представления исследовательского пространства как динамического единства деятельностных и предметных отношений математического моделирования исследовательского умения сетями Петри [ 7; 8 ].

В целом, можно определить многоуровневую ячейку цикла сети Петри. На первом базисном уровне ячейка сети Петри определяет взаимосвязь базисных отношений: система исследовательских умений (ориентировочный базисный образ теории); исследовательская сеть Петри (средства исполнительского процесса реализации исследовательского знания) и обобщённые исследовательские умения (результат и контрольно-базисный компонент исследовательской деятельности). 


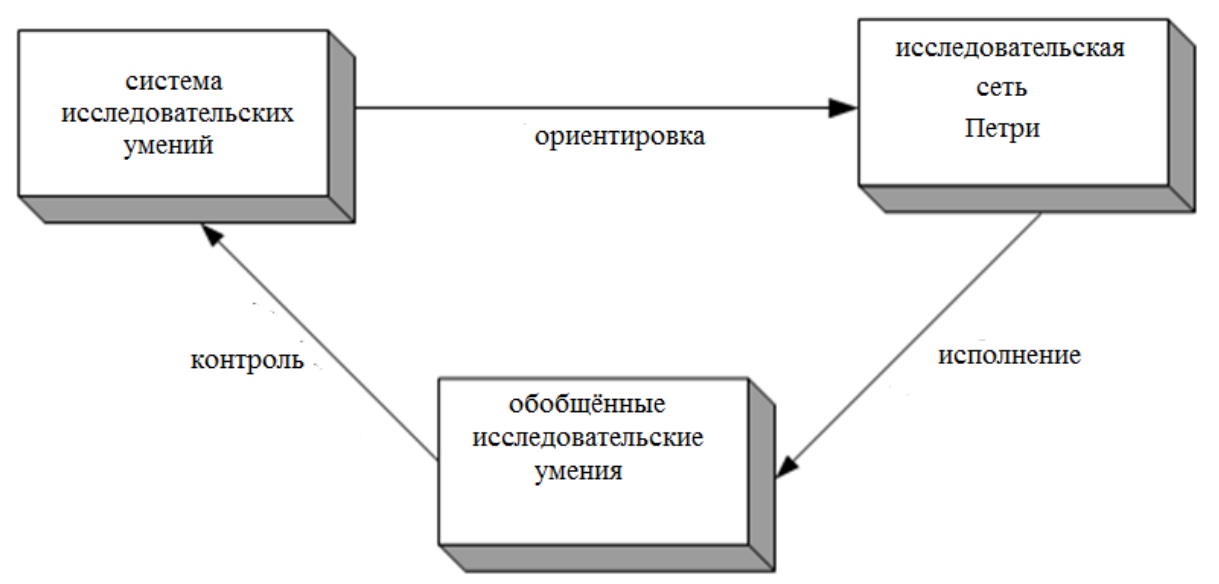

Рисунок 1 - Первый базисный уровень сетей Петри для моделирования и анализа исследовательских умений.

Второй базисный уровень сети Петри надо рассматривать как соотношение между предметными и деятельностными компонентами процесса организации формирования исследовательских умений. Предметными условиями являются: личностные особенности учащегося (1); средства исследовательской деятельности (2); исследовательский предмет процесса анализа явления (3); результат исследовательского процесса (4) и прогнозный элемент развития исследовательского умения (5). В зависимости от глобальности процесса количество предметных условий может изменяться.

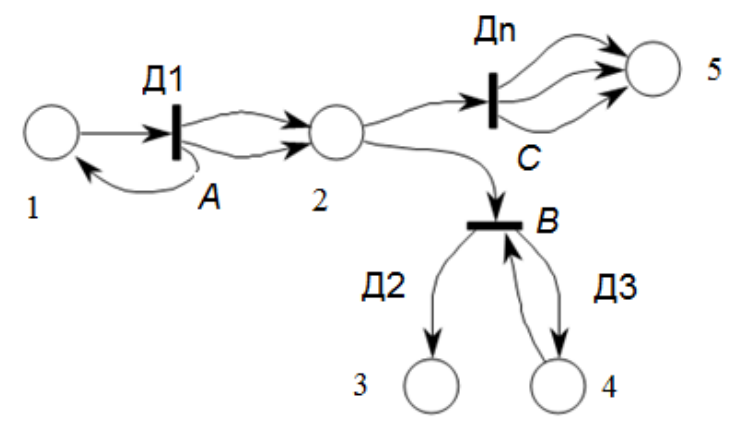

Рисунок 2 - Второй базисный уровень сетей Петри для моделирования и анализа исследовательских умений.

Деятельностные компоненты процесса исследовательского умения на интегральные базисные представления структуры исследовательской деятельности относительно: обобщенного процесса исследовательского умения (Д1); специфической технологии процесса исследовательского умения (Д2); контрольной технологии процесса исследовательского умения (Д3); прогнозная технологии процесса исследовательского умения (Ди).

В процессе развития деятельностных компонентов возникают многофазные процессы, которые отражают точки мгновенного принятия разноплановых решений. Это позволяет проектировать многосвязные деятельностные исследовательские процессы.
Фазовыми точками являются моменты деятельностей (А; В; С ...), которые создают фазово-временное пространство исследовательских умений [ 9; 10 ].

Третий базисный уровень сети Петри надо рассматривать как соотношение между предметными и деятельностными компонентами процесса организации формирования исследовательских умений относительно генетических дидактикоматематических элементов.

$$
\begin{aligned}
& \text { 1) } Z(X)=3 x_{1}+2 x_{2} \rightarrow \max \\
& \left\{\begin{array}{l}
2 x_{1}+2 x_{2} \leq 210, \\
2 x_{1}+x_{2} \leq 100,
\end{array}\right. \\
& x_{1} \geq 0, x_{2} \geq 0 .
\end{aligned}
$$




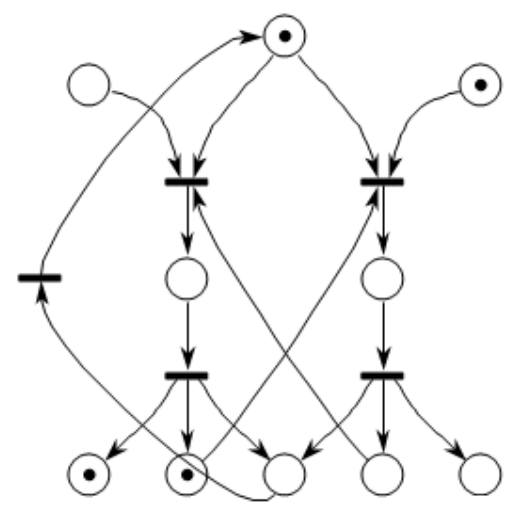

\section{Рисунок 3 - Третий базисный уровень сетей Петри для моделирования и анализа исследовательских умений.}

На данном уровне выделяются функциональные отношения между элементами исследовательского предмета, которые преобразуются в предметно-деятельностные отношения функционально-операционных моментов оперативных схем исследовательского мышления, моделирующих сетями Петри.
Дальнейшее применение сетей Петри для формирования обобщённых исследовательских умений связывается с разработкой базисных компонентов предметно-деятельностных отношений на уровне автоматизированных компьютерных обучающих комплексов.

\section{References:}

1. Mishchik SA (2014) Pedagogometrika and mathematical modeling educational activity. Materialy Mezhdunarodnoy nauchnoy konferenctsii "Modern mathematics in science" - 30.06.2014. ISJ Theoretical \&Applied Science 6(14): 54-56 Caracas, Venezuela. doi: http://dx.doi.org/10.15863/TAS.2014.06.14.10

2. Mishchik SA (2014) Simulation training activity methods of mathematical logic. Materialy Mezhdunarodnoy nauchnoy konferenctsii "Eurapean Science and Education" - 30.07.2014. ISJ Theoretical \&Applied Science 7(15): 72-74 Marseille, France. doi: http://dx.doi.org/10.15863/TAS.2014.07.15.13

3. Mishchik SA (2014) Mathematical modeling system integrity-cycle of life activity - first goal pedagogometriki. Materialy Mezhdunarodnoy nauchnoy konferenctsii "European Applied Sciences" - 30.08.2014. ISJ Theoretical \&Applied Science 8(16): 77-79 Aix-enProvence, France. doi: http://dx.doi.org/10.15863/TAS.2014.08.16.13

4. Mishchik SA (2014) Mathematical modeling system integrity-curricular activities - the second problem pedagogometriki. Materialy Mezhdunarodnoy nauchnoy konferenctsii "European Innovation" - 30.09.2014. ISJ ISPC European Research, Birmingham, United Kingdom
Theoretical \&Applied Science 9(17): 126-128 Martigues, France. doi: http://dx.doi.org/10.15863/TAS.2014.09.17.21

5. Mishchik SA (2014) Mathematical modeling holistic-systemic communicative activity - the third task pedagogometriki. Materialy Mezhdunarodnoy nauchnoy konferenctsii "European Scientific Achievements" 30.10.2014. ISJ Theoretical \&Applied Science 10(18): 45-47 Brighton, UK. doi: http://dx.doi.org/10.15863/TAS.2014.10.18.11

6. Mishchik SA (2014) Mathematical modeling integrity - system performance subject - fourth task pedagogometriki. Materialy Mezhdunarodnoy nauchnoy konferenctsii "Eurapean Science and Technology" 30.11.2014. ISJ Theoretical \&Applied Science 11(19): 51-54 Southampton, United Kingdom. doi:

http://dx.doi.org/10.15863/TAS.2014.11.19.10

7. Mishchik SA (2014) Tselostno-sistemnyy tsikl uchebnoy zhiznedeyatel'nosti - model' professional'noy deyatel'nosti shirokoprofil'nogo spetsialista V sbornike: Deyatel'nostnaya teoriya ucheniya: sovremennoe sostoyanie i perspektivy. Materialy Mezhdunarodnoy nauchnoy konferentsii. Otvetstvennye redaktory: Yu.P. 
Zinchenko, O.A. Karabanova, A.I. Podol'skiy, G.A. Glotova. Moscow, pp. 352-354.

8. Tokmazov GV (2014) Matematicheskoe modelirovanie v uchebno-professional'noy deyatel'nosti. Materialy Mezhdunarodnoy nauchnoy konferenctsii "Modern mathematics in science" - 30.06.2014. ISJ Theoretical \&Applied Science 6(14): 44-46 Caracas, Venezuela. doi: http://dx.doi.org/10.15863/TAS.2014.06.14.8

9. Tokmazov GV (2014) Analysis says study skills in the study of mathematics. Materialy Mezhdunarodnoy nauchnoy konferenctsii "Eurapean Science and Education" 30.07.2014. ISJ Theoretical \&Applied Science 7(15): 72-74 Marseille, France. doi: http://dx.doi.org/10.15863/TAS.2014.07.15.14

10. Tokmazov GV (2014) Mathematical modeling research skills in educational activity methods of probability theory. Materialy Mezhdunarodnoy nauchnoy konferenctsii "Eurapean Science and Technology" 30.11.2014. ISJ Theoretical \&Applied Science 11(20): 66-69 Southampton, United Kingdom. doi:

http://dx.doi.org/10.15863/TAS.2014.11.19.13
11. Tokmazov GV (1994) Zadachi dinamicheskogo kharaktera. Matematika V shkole. No. 5, pp.9-12.

12. Tokmazov GV (1999) Ukrupnenie didakticheskikh edinits $\mathrm{v}$ zadachakh po teorii veroyatnostey. Matematika v shkole. No.4. pp.81-85.

13. Tokmazov GV (1999) Model' formirovaniya issledovatel'skikh umeniy na osnove trekhkomponentnoy strukture umstvennogo deystviya. Nauchnye trudy Moskovskogo pedagogicheskogo gosudarstvennogo universiteta. Seriya: Estestvennye nauki. Moscow, Prometey, pp.68-88.

14. Tokmazov GV (2013) Sistematizatsiya i differentsirovannyy podkhod pri obuchenii resheniyu zadach nachal teorii veroyatnostey: monografiya. - 2-e izd., dop.i isprav. Novorossiysk: GMU imeni admirala F.F.Ushakova, 176. ISBN 978-5-89426-071-6

15. Tokmazov GV (2014) Opredelenie usloviy podgotovki uchashchikhsya $\mathrm{k}$ organizatsii issledovatel'skikh umeniy pri obuchenii matematike. Kazanskaya nauka. No. 7. pp. 180185 . 